\title{
Kinetic coefficients of semiconductor superlattices in high-frequency electromagnetic fields
}

\author{
A. V. Shorokhov ${ }^{1}$, N.S. Prudskikh ${ }^{1}$, M. B. Semenov ${ }^{2}$, V. D. Krevchik ${ }^{2}$, \\ M. A. Pyataev ${ }^{1}$, S. E. Golovatyuk ${ }^{1}$, Tian-Rong Li ${ }^{3}$, Yu-Hua Wang ${ }^{3}$ \\ ${ }^{1}$ National Research Mordovia State University, Bolshevistskaya, 68, Saransk, 430005, Russia \\ ${ }^{2}$ Penza State University, Krasnaya, 40, Penza, 440026, Russia \\ ${ }^{3}$ Institute of Functional and Environmental Materials, Lanzhou University, Lanzhou, China \\ alex.shorokhov@mail.ru
}

PACS 73.63.-b

DOI 10.17586/2220-8054-2017-8-6-740-745

\begin{abstract}
Kinetic coefficients of semiconductor superlattice are obtained from the Boltzmann transport equation with Bhatnagar-Gross-Krook (BGK) collision term and Poisson equation. Using the universal analytic procedure, we found kinetic coefficient in the quasistatic limit starting from the exact solution of the Boltzmann transport equation. It is shown that the Einstein relation for the diffusion coefficient is applicable only for weak fields and it is not valid in the general case. As a consequence, a drift-diffusion model of miniband transport in the case of strong dc and ac fields should be corrected, taking into account the kinetic coefficients obtained from the Boltzmann equation.
\end{abstract}

Keywords: superlattice, Boltzmann equation, drift-diffusion model, THz radiation, diffusion coefficient, drift velocity, Maxwell frequency, Einstein relation.

Received: 13 November 2017

Revised: 29 November 2017

\section{Introduction}

The problem of electric stability for a semiconductor superlattice is a crucial one for the practical realization of sub-THz- and THz-based devices. For example, it is well-known that the development of instabilities and the formation of electric domains in a superlattice placed in a dc electric field leads to the destruction of the $\mathrm{THz}$ gain [1]. Therefore, the main problem in the realization of superlattice-based $\mathrm{THz}$ devices is finding operational conditions which simultaneously allow one to achieve gain at $\mathrm{THz}$ frequencies and to avoid destructive spacecharge instabilities. Correct description of instability effects requires taking into account spatial-dispersion effects. Such approach for the case of dc biased superlattice was formulated by Ignatov and Shashkin [1-3] and Bonilla et al. [4-6]. As a rule, a drift-diffusion model is used to describe transport and high-frequency properties of superlattices in the quasistatic case. In particular, this model allows taking into account spatial-dispersion effects. However, as it will be shown below, this model can give incorrect results because the correct dependence of kinetic coefficients on the amplitudes of $\mathrm{dc}$ and ac fields as well as temperature in the quasistatic case can be obtained only from the Boltzmann and Poisson equations. Note that the analysis of kinetic coefficients of superlattice can give the important information about spatial-dispersion effects and instabilities by analogy with the Gunn effect.

In this paper, we present an approach from which general expressions for the field-dependent average drift velocity, Maxwell frequency and diffusion coefficient are derived starting from the exact solution of Boltzmann equation. As a rule the diffusion coefficient is obtained from the Einstein relation in the framework of driftdiffusion model. However the Einstein relation is not always applicable for strong fields [5,7]. In connection with it, the calculation of the diffusion coefficient is an important separate question. In this paper we obtain the diffusion coefficient from the exact solution of Boltzmann equation and show that it is sufficiently different from the diffusion coefficient obtained from the Einstein relation.

Let us consider a superlattice under the action of the dc field $E_{0}$ directed along the $x$-axis and the strong ac field $E_{1} \cos \left(\omega_{1} t\right)$. Our main goal is to find and analyze the kinetic coefficients of semiconductor superlattice in quasi-static approximation starting from the exact solution of the kinetic equation and taking into account spatial-dispersion effects connected with a small perturbation $E_{2} \cos \left(\omega_{2} t-k_{2} x\right)$.

In the following, we will use the standard dispersion relation in the tight-binding approximation:

$$
\varepsilon(p)=\frac{\Delta}{2}\left[1-\cos \left(\frac{p d}{\hbar}\right)\right],
$$

where $d$ is the period of a superlattice, $\Delta$ is the miniband width, $p$ is the quasimomentum. 


\section{Boltzmann equation}

In this paper, we will use the traditional method for calculating a high-frequency current based on the use of kinetic equation for miniband electrons with BGK (Bhatnagar-Gross-Krook) collision integral, which permits adequate allowance for the particle-number conservation law for scattering in inhomogeneous field and, as a consequence, makes it possible to take into account the influence of the carrier drift and diffusion effects on the space-charge wave spectrum:

$$
\frac{\partial f}{\partial t}+V(p) \frac{\partial f}{\partial x}+e E(x, t) \frac{\partial f}{\partial p}=-\frac{1}{\tau}\left(f-\frac{n(x)}{n_{0}} f_{0}\right),
$$

where $V(p)=V_{0} \sin (p d / \hbar), V_{0}=d \Delta / 2 \hbar$ is the maximum electron velocity in the miniband, $E(x, t)=E_{0}+$ $E_{1} \cos \left(\omega_{1} t\right)+E_{2} \cos \left(\omega_{2} t-k_{2} x\right)$ is the total electric field, $n(x)$ is the electron density, $f_{0}=\left(n_{0} d / 2 \pi \hbar I_{0}\right)$ $\exp [(\Delta / 2 T) \cos (p d / \hbar)]$ is the equilibrium distribution function normalized to the equilibrium electron density $n_{0}, I_{m}(m \in \mathbb{Z})$ is the modified Bessel function of argument $\Delta / 2 T, \tau$ is the relaxation time, $T$ is the lattice temperature.

The electron distribution functions, due to the periodicity of the quasimomentum, permit representation in the form of a Fourier series:

$$
f(p)=\sum_{m=-\infty}^{\infty} f_{m}(x) e^{i m \varphi}, \quad f_{0}(p)=\sum_{m=-\infty}^{\infty} f_{m}^{0} e^{i m \varphi},
$$

where $\varphi=p d / \hbar, f_{m}^{0}=n_{0} d I_{m} / 2 \pi \hbar I_{0}$.

In its turn, $f_{m}(x)$ as well as $n(x)$ permits in the segment $0<x<L$ ( $L$ is the superlattice lenght) a representation in the form of the following Fourier series:

$$
f_{m}(x)=\sum_{s=-\infty}^{\infty} f_{m s} e^{i k x}, \quad n(x)=\sum_{s=-\infty}^{\infty} n_{s} e^{i k x}
$$

where $k=2 \pi s / L$ is the wave number, $s \in \mathbb{Z}$.

Substituting (3) and (4) into (2), we get:

$$
\frac{\partial f_{m s}}{\partial t}+i k V(\varphi) f_{m s}+\frac{e d}{\hbar} E(x, t) i m f_{m s}+\nu f_{m s}=\nu \frac{n_{s}}{n_{0}} f_{m}^{0} .
$$

To find $n_{s}(t)$ we need to use the Poisson equation:

$$
\frac{\partial E(x, t)}{\partial x}=\frac{4 \pi e}{\varepsilon}\left(n(x)-n_{0}\right)
$$

where $\varepsilon$ is the lattice dielectric constant.

As a result we get the exact solution of the kinetic equation (2) in the form:

$$
\begin{gathered}
f=\sum_{m=-\infty}^{\infty} f_{m}^{0} \sum_{l_{1}, l_{2}=-\infty}^{\infty} \sum_{\nu_{1}, \nu_{2}=-\infty}^{\infty} J_{l_{1}}\left(m \beta_{1}\right) J_{l_{2}}\left(m \beta_{2}\right) J_{l_{1}+\nu_{1}}\left(m \beta_{1}\right) J_{l_{2}+\nu_{2}}\left(m \beta_{2}\right) \times \\
\times\left[\frac { \varepsilon k _ { 2 } E _ { 2 } } { 8 i \pi e n _ { 0 } } \left(\frac{\exp \left[-i\left(\nu_{1} \omega_{1}+\left(\nu_{2}-1\right) \omega_{2}\right) t\right] \exp \left(-i k_{2} x\right)}{-i k_{2} \tau V(\varphi)+1+i m \Omega_{0} \tau+i\left(l_{1} \omega_{1}+\left(l_{2}+1\right) \omega_{2}\right) \tau}-\right.\right. \\
\left.\quad-\frac{\exp \left[-i\left(\nu_{1} \omega_{1}+\left(\nu_{2}+1\right) \omega_{2}\right) t\right] \exp \left(i k_{2} x\right)}{i k_{2} \tau V(\varphi)+1+i m \Omega_{0} \tau+i\left(l_{1} \omega_{1}+\left(l_{2}-1\right) \omega_{2}\right) \tau}\right)+ \\
\left.+\frac{\exp \left[i\left(\nu_{1} \omega_{1}+\nu_{2} \omega_{2}\right) t\right]}{1+i m \Omega_{0} \tau+i\left(l_{1} \omega_{1}+l_{2} \omega_{2}\right) \tau}\right] \exp \left[i\left(\nu k_{2} x+m \varphi\right)\right]
\end{gathered}
$$

Here $\nu=1 / \tau, \beta_{i}=\Omega_{i} / \omega_{i}(i=1,2), \Omega_{j}=e d E_{j} / \hbar(j=0,1,2), J_{l}(\beta)$ is the Bessel function.

Using the distribution function (7), one can find the average electron velocity:

$$
V_{a}(t)=\langle V(\varphi)\rangle_{\varphi}=\frac{\hbar}{d} \int_{0}^{2 \pi} V(\varphi) f(\varphi) d \varphi
$$

and the average complex current on the frequency $\omega_{2}$

$$
j=2 e\left\langle V_{a}(t) \exp \left[i\left(\omega_{2} t-k_{2} x\right)\right]\right\rangle_{t},
$$



current:

In the case of a small perturbation $\beta_{2} \ll 1$ and a small $k_{2}$, we get the following expression for the complex

$$
\begin{gathered}
j=-\frac{\varepsilon k_{2} E_{2} V_{0} I_{1}}{8 \pi I_{0}} \sum_{l_{1}=-\infty}^{\infty} J_{l_{1}}^{2}\left(\beta_{1}\right)\left[\frac{1-i\left(l_{1} \omega_{1}-\omega_{2}+\Omega_{0}\right) \tau}{1+\left(l_{1} \omega_{1}-\omega_{2}+\Omega_{0}\right)^{2} \tau^{2}}-\frac{1-i\left(l_{1} \omega_{1}-\omega_{2}-\Omega_{0}\right) \tau}{1+\left(l_{1} \omega_{1}-\omega_{2}-\Omega_{0}\right)^{2} \tau^{2}}\right]+ \\
+\frac{\varepsilon k_{2}^{2} E_{2} V_{0}^{2} \tau}{16 \pi}\left[2 \frac{\left(1+i \omega_{2} \tau\right)^{2}}{\left(1+\omega_{2}^{2} \tau^{2}\right)^{2}}-\frac{I_{2}}{I_{0}} \sum_{l_{1}=-\infty}^{\infty} J_{l_{1}}^{2}\left(2 \beta_{1}\right) \times\right. \\
\left.\times\left(\frac{\left(1-i\left(l_{1} \omega_{1}-\omega_{2}+2 \Omega_{0}\right) \tau\right)^{2}}{\left(1+\left(l_{1} \omega_{1}-\omega_{2}+2 \Omega_{0}\right)^{2} \tau^{2}\right)^{2}}+\frac{\left(1-i\left(l_{1} \omega_{1}-\omega_{2}-2 \Omega_{0}\right) \tau\right)^{2}}{\left(1+\left(l_{1} \omega_{1}-\omega_{2}-2 \Omega_{0}\right)^{2} \tau^{2}\right)^{2}}\right)\right]+ \\
+\frac{i e^{2} d n_{0} V_{0} I_{1} E_{2}}{2 I_{0} \hbar} \sum_{l_{1}=-\infty}^{\infty} J_{l_{1}}^{2}\left(\beta_{1}\right)\left[-\frac{2}{1+\left(l_{1} \omega_{1}+\Omega_{0}\right)^{2} \tau^{2}}+\right. \\
\left.+\frac{1-i\left(l_{1} \omega_{1}+\omega_{2}+\Omega_{0}\right) \tau}{1+\left(l_{1} \omega_{1}+\omega_{2}+\Omega_{0}\right)^{2} \tau^{2}}+\frac{1-i\left(l_{1} \omega_{1}+\omega_{2}-\Omega_{0}\right) \tau}{1+\left(l_{1} \omega_{1}+\omega_{2}-\Omega_{0}\right)^{2} \tau^{2}}\right] .
\end{gathered}
$$

\section{Quasistatic limit}

To find the quasistatic limit of the current we have to impose the additional limitation on $\omega_{1}$ and $\omega_{2}: \omega_{1} \tau \ll 1$, $\omega_{2} \tau \ll 1$. Using the saddle-point method [8,9] we get the formula for the current in quasistatic limit as a power series in $k_{2}$ :

$$
j=\frac{\varepsilon}{4 \pi} E_{2}\left(\omega_{m}+i k_{2} V_{d}+k_{2}^{2} D\right)
$$

In formula (11), the drift velocity can be written as [10]

$$
V_{d}=\frac{V_{p} I_{1}}{\pi I_{0}} \int_{0}^{2 \pi} I^{E T}\left(\Omega_{1} \cos \theta+\Omega_{0}\right) d \theta
$$

where:

$$
I^{E T}(\omega)=\frac{\omega \tau}{1+\omega^{2} \tau^{2}}
$$

is the Esaki-Tsu characteristic, and $V_{p}=V_{0} / 2$ is the peak drift velocity.

The dependence of $V_{d}$ on $E_{0}$ is shown on Fig. 1. The ratio of Bessel functions in (12) takes into account the effect connected with the thermal distribution of miniband electron. It follows from Eq. (12) that the effect of temperature leads to a reduction of the drift velocity. The growth of amplitude of ac field leads to a shift of the maximum of the dependence of the drift velocity on the dc bias to the region of higher static fields and reduction of the drift velocity (Fig. 1).

The differential Maxwell frequency is expressed in terms of the drift velocity as:

$$
\omega_{m}=\frac{4 \pi e n_{0}}{\varepsilon} \frac{d V_{d}}{d E_{0}} .
$$

The dependence of Maxwell frequency on the dc bias is shown on Fig. 2 at the different values of ac field. The diffusion coefficient has the following form:

$$
D=\frac{V_{0}^{2} \tau}{4}\left\{2-\frac{I_{2}}{4 \pi \tau I_{0}} \frac{\partial}{\partial \Omega_{0}} \int_{0}^{2 \pi}\left[I^{E T}\left(2 \Omega_{1} \cos \theta+2 \Omega_{0}\right)-I^{E T}\left(2 \Omega_{1} \cos \theta-2 \Omega_{0}\right)\right] d \theta\right\} .
$$

Note that the Einstein relation $D(E)=T V_{d}(E) / e E$ is not valid in this case. In connection with it, the use of the Einstein relation is incorrect for systems with strong dc and ac fields, except in some particular cases.

In the limit of the pure dc field $E_{1}=0$, we obtain for the Maxwell frequency:

$$
\omega_{m}=\omega_{p}^{2} \tau, \quad N^{E T}\left(\Omega_{0}\right)=\omega_{p}^{2} \frac{\partial I^{E T}\left(\Omega_{0}\right)}{\partial \Omega_{0}},
$$

for the drift velocity $[11,12]$ (this dependence is in a good agreement with experimental data [13]):

$$
V_{d}=2 V_{p} \frac{I_{1}}{I_{0}} I^{E T}\left(\Omega_{0}\right),
$$




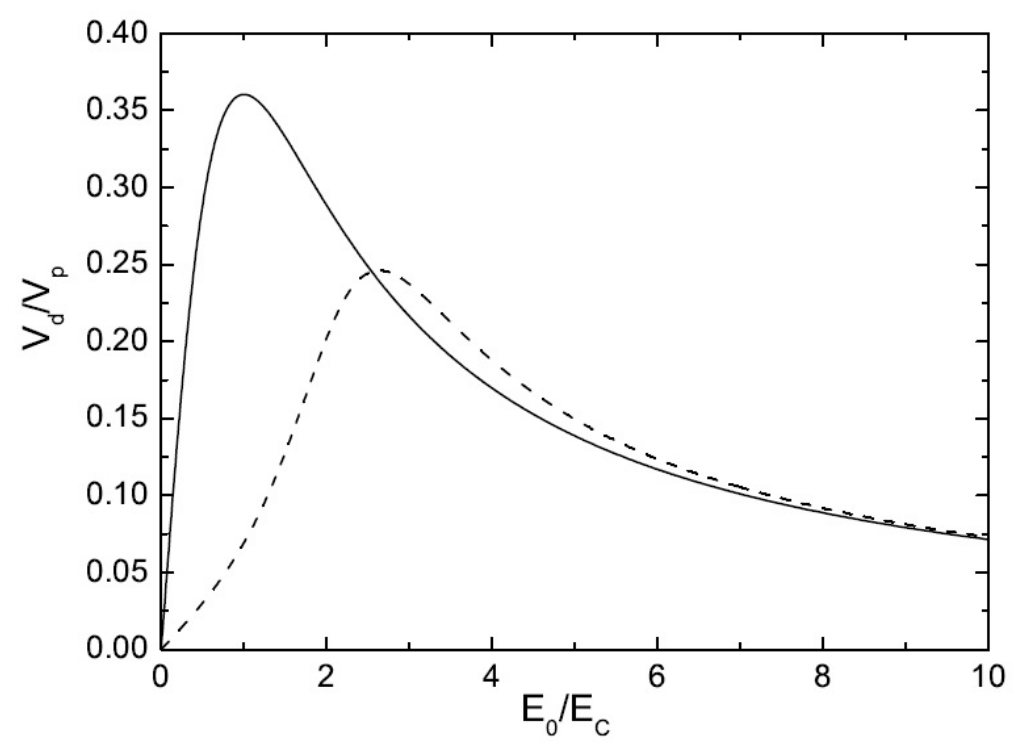

FIG. 1. The dependence of the drift velocity on the dc bias $E_{0}$ at $T=300 \mathrm{~K}$. Solid line corresponds to $E_{1}=0$, the dash line corresponds to $E_{1} \tau=2.1$. $E_{c}=\hbar / e d \tau\left(\Omega_{0} \tau=E_{0} / E_{c}\right)$ is the critical field corresponding to the maximum of Esaki-Tsu I-V characteristic at $E_{1}=0$

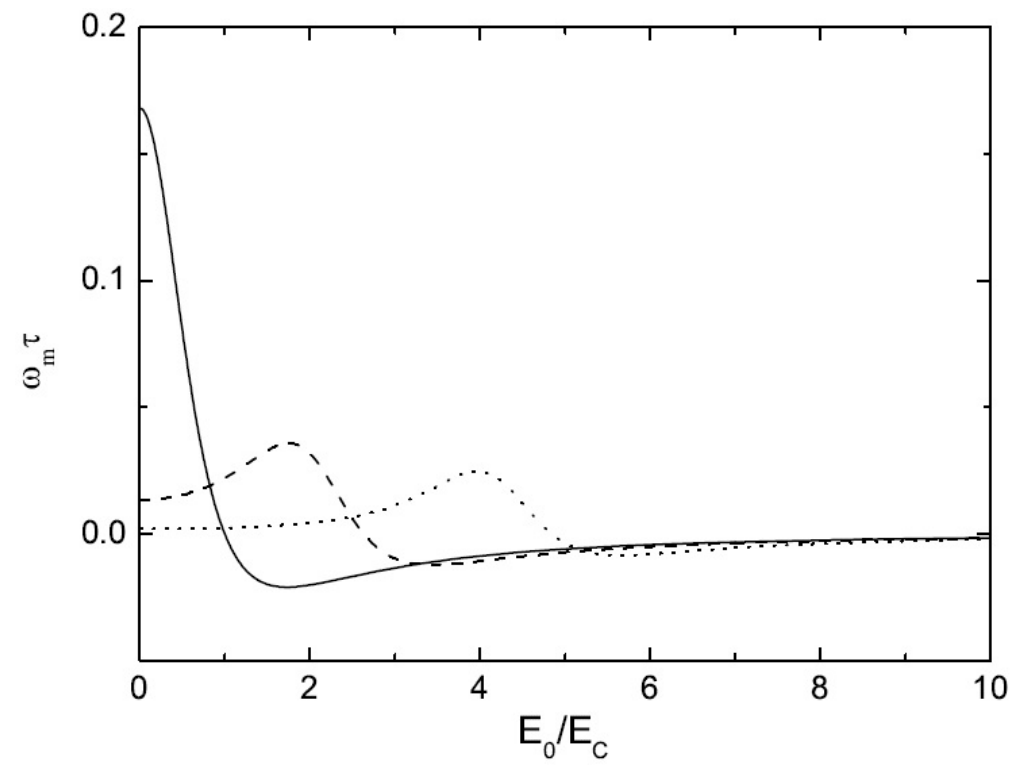

FIG. 2. The dependence of the Maxwell frequency on the dc bias $E_{0}$ at $T=300 \mathrm{~K}$. Solid line corresponds to $E_{1}=0$, the dash line corresponds to $E_{1} \tau=2.1$, the dot line corresponds to $E_{1} \tau=4.3 . \tau=200 \mathrm{fs}, \Delta=40 \mathrm{meV}, n_{0}=0.5 \times 10^{16} \mathrm{~cm}^{-3}, d=6 \mathrm{~nm}$

and for the diffusion coefficient:

$$
D=\frac{V_{0}^{2} \tau}{4}\left[1-\frac{I_{2}}{I_{0}} N^{E T}\left(2 \Omega_{0}\right)\right]
$$

Here:

$$
N^{E T}(\omega)=\frac{1-\omega^{2} \tau^{2}}{\left(1+\omega^{2} \tau^{2}\right)^{2}} .
$$

The difference between the diffusion coefficients obtained from the Boltzmann equation and from the Einstein relation is shown on Figs. 3 and 4 for the case of the pure dc field. One can see that the Einstein relation gives the correct result only in the case of small dc fields $\Omega_{0} \tau<0.1$. At the same time, the diffusion coefficient obtained 


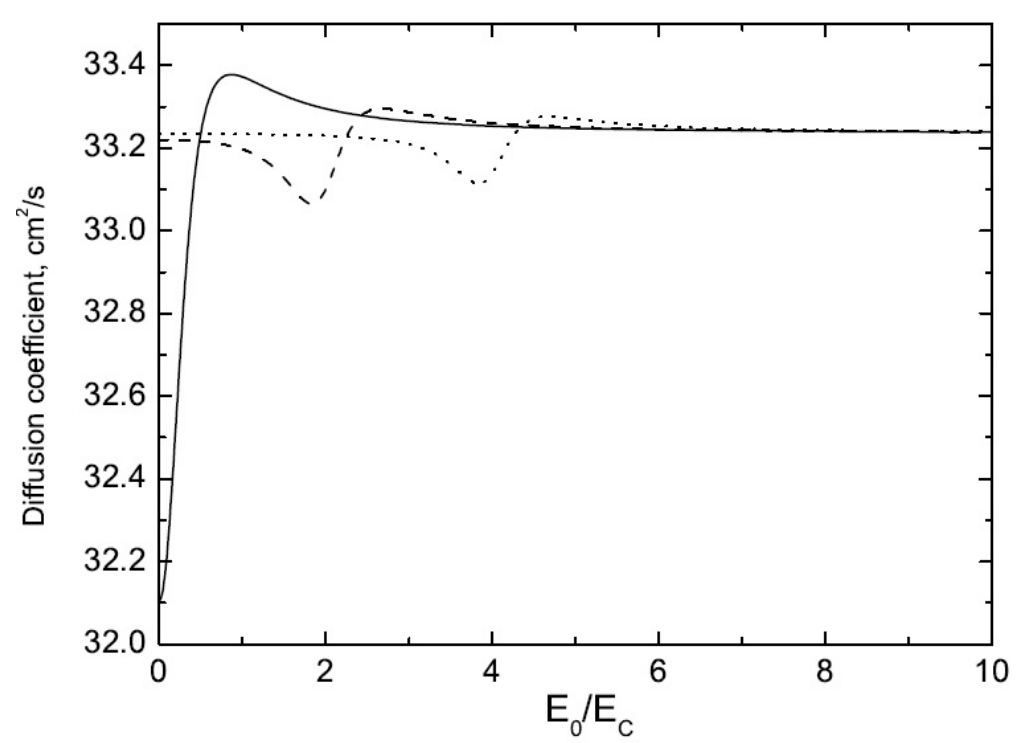

FIG. 3. The dependence of the diffusion coefficient on the dc bias $E_{0}$ at $T=300 \mathrm{~K}$. Solid line corresponds to $E_{1}=0$, the dash line corresponds to $E_{1} \tau=2.0$, the dot line corresponds to $E_{1} \tau=4.0$. The other parameters are the same as in Fig. 2

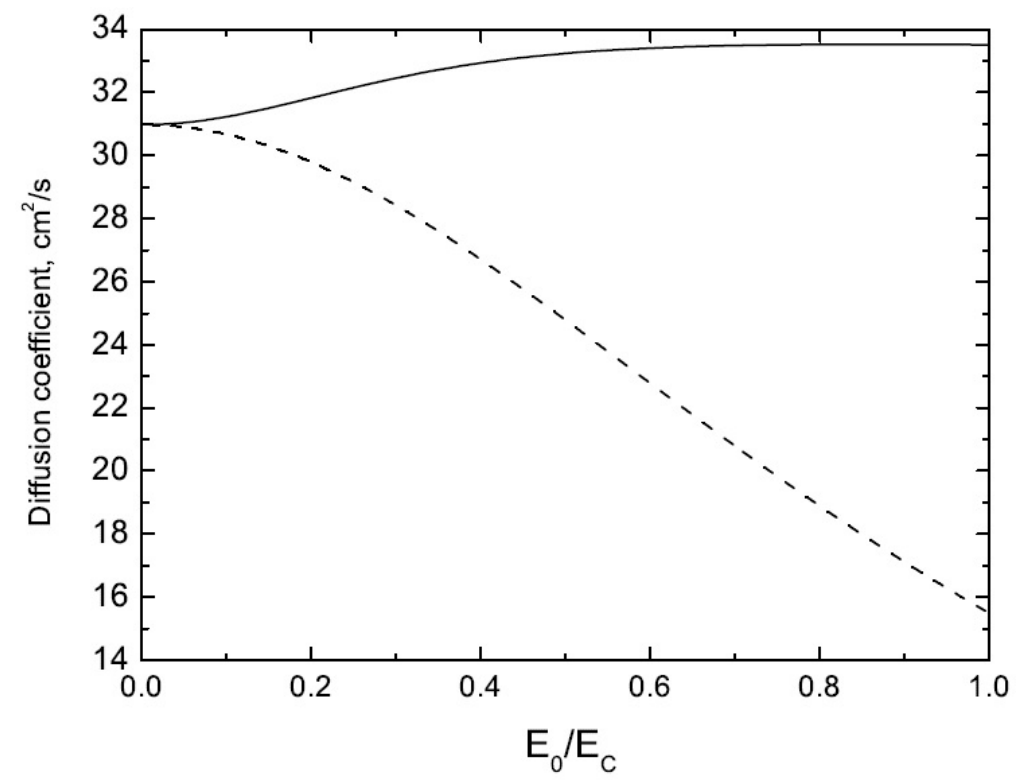

FIG. 4. The dependence of the diffusion coefficient on the dc bias $E_{0}$ at $T=300 \mathrm{~K}$. Solid line corresponds to the diffusion coefficient calculated by formula (18), the dash line corresponds to the diffusion coefficient calculated by the Einstein relation. The other parameters are the same as in Fig. 2 
from the Boltzmann equation increases with an increase in the dc field, while the diffusion coefficient obtained from the Einstein relation decreases with the increasing of dc field.

In the opposite case of the ac pump field $\left(E_{0}=0\right)$, the kinetic coefficients have the form:

$$
\begin{gathered}
\omega_{m}=\frac{\omega_{p}^{2} \tau}{\left(1+\Omega_{1}^{2} \tau^{2}\right)^{3 / 2}}, \\
V_{d}=0, \\
D=\frac{V_{0}^{2} \tau}{2}\left[1-\frac{I_{2}}{I_{0}} \frac{1}{\left(1+4 \Omega_{1}^{2} \tau^{2}\right)^{3 / 2}}\right] .
\end{gathered}
$$

\section{Conclusion}

We have modified the drift-diffusion model for the semiconductor superlattice using the exact solution of the Boltzmann equation. The obtained kinetic coefficient such as the Maxwell frequency, drift velocity and diffusion coefficient differ from the common coefficients of standard drift-diffusion model. In particular, the Maxwell frequency and drift velocity has the additional temperature factor though the relationship between these coefficients are the same as in the drift-diffusion model. At the same time, the diffusion coefficient differs drastically from that of the drift-diffusion model, and as a consequence, the Einstein relationship is not valid in this case.

\section{Acknowledgements}

The authors would like to thank Kirill Alekseev for collaboration and useful ideas. This work was supported by the Ministry of Education and Science of the Russian Federation (Project No. 3.6321.2017/8.9), and RFBR (Project No. 17-02-00969).

\section{References}

[1] Ignatov A. A., Shashkin V. I. Bloch oscillation of electrons and instabilities of space-charge waves in semiconductor superlattices. Sov. Phys. JETP, 1987, 66, P. 526-530.

[2] Ignatov A. A., Shashkin V. I. Diffusion coefficient of heated carries, spectrum of space-charge waves, and characteristic instability frequencies of semiconductor superlattices. Sov. Phys. Semicond, 1984, 18, P. 721-724.

[3] Ignatov A. A., Shashkin V.I. Quasihydrodynamdice description of the instability of space charge waves and impedance of bounded superlattice structures. Sov. Phys. Semicond, 1987, 21, P. 973-976.

[4] Bonilla L. L., Alvaro M., Carretero M. Theory of spatially inhomogeneous Bloch oscillations in semiconductor superlattices. Physical Review B, 84, 2011, P. 155316 1-17.

[5] Bonilla L.L., Escobedo R., Perales Á. Generalized drift-diffusion model for miniband superlattices. Physical Review B, 2003, 68, P. 2413041-4(R).

[6] lvaro M., Carretero M., Bonilla L. L. Numerical method for hydrodynamic modulation equations describing Bloch oscillations in semiconductor superlattices. Journal of Computational Physics, 2012, 231, P. 4499-4514.

[7] Bryksin V.V., Kleinert P. Theory of quantum diffusion in biased semiconductors. J. Phys.: Condens. Matter, 2003,15, P. $1415-1425$.

[8] Shorokhov A. V., Aleksev K. N. High-frequency absorption and gain in superlattices: Semiquasistatic approach. Physica E, 2006, 33, P. 284-295.

[9] Shorokhov A. V., Aleksev K. N. Quantum Derivatives and Terahertz Gain in a Superlattice. JETP, 2006, 105, P. 198-200.

[10] Winnerl S., Schomburg E., Grenzer J., Regl H.-J., Ignatov A. A., Semenov A. D., Renk K. F., Pavel'ev D. G., Koschurinov Yu., Melzer B., Ustinov V., Ivanov S., Schaposchnikov S., Kop'ev P. S. Quasistatic and dynamic interaction of high-frequency fields with miniband electrons in semiconductor superlattices. Physical Review B, 1997, 56, P. 10303-10307.

[11] Suris R. A. and Shchamkhalova B. S. Heating of electrons in superlattice semiconductors. Sov. Phys. Semicond, 1984, 18, P. 738-742.

[12] Ignatov A. A., Dodin E. P., Shashkin V. I. Transient response theory of semiconductor superlattices: connection with Bloch oscillations Mod. Phys. Lett. B, 1991, 5, P. 1087-1094.

[13] Grahn H. T., von Klitzing K., Ploog K., Dohler G.H. Electrical transport in narrow-miniband semiconductor superlattices Phys. Rev. B, 1991, 43, P. 12094-12097. 